\title{
UV Dichroism in Vertically Aligned Nematic Displays
}

\author{
N. Bennis , B. Cerrolaza , D. P. Medialdea , J. M. Otón , \\ P. Kula , and R. Dąbrowski \\ Universidad Politécnica de Madrid (UPM), Ciudad Universitaria, \\ Madrid, Spain \\ Military University of Technology, Warsaw, Poland
}

\begin{abstract}
Vertically aligned negative nematics are employed in high-end projection applications, where extremely high light fluxes are employed. Under these conditions, the UV photochemical stability of the material becomes an issue that must be included in the characterization process of such devices. Photochemical aging tests for vertically aligned microdisplay, however, must take into account the linear dichroism shown by the liquid crystal material upon switching. In this work a method to evaluate UV linear dichroism is described. The method is included in a comparative study of the electrooptical performance of two negative nematic mixtures, one experimental and one commercial.
\end{abstract}

Keywords: electrooptical response; liquid crystals; UV dichroism; vertically aligned nematic

\section{INTRODUCTION}

Homeotropically aligned nematics having negative dielectric anisotropy are being increasingly used for high-end display applications, both in direct view and in projection devices Vertically aligned negative nematic (VAN) liquid crystal materials feature high contrast and excellent response time making them the preferred choice for high resolution projectors. In these devices, VANs are customarily associated to an active matrix silicon to produce liquid crystal on silicon (LCOS) reflective devices These projectors, 
employed in large-area displays and digital cinema, must withstand extremely large light fluxes from high power lamps. The spectrum of these lamps may contain a substantial fraction of ultraviolet light, which might be harmful for the LC material [4], speeding up the material aging and eventually jeopardizing the lifetime of the LCOS light engine.

To avoid UV photochemical degradation, a number of steps are included in the manufacturing process of VAN LCOS devices. Inorganic alignment surfaces prepared by angular thermal vacuum deposition of inorganic oxides are usually chosen instead of organic alignment films. UV cut-off filters are customarily inserted in the light path. The cut-off wavelength is located in the $400-420 \mathrm{~nm}$ region to avoid degradation of the color gamut. Nevertheless a residual fraction of UV radiation impinges on the LC material. Even a small UV fraction may produce significant damage in the material via radical formation that triggers photodegradation of the $\mathrm{LC}$ This degradation causes deterioration of physical and chemical properties, increasing of conductivity in liquid crystals. Therefore UV absorption must be taken into account as a design parameter for material selection in these applications.

On the other hand, the LC birefringence is linked by the Kramers-Krönig relationship to linear dichroism in the electronic absorption spectrum. Dichroism implies that the absorption of UV polarized light depends on the relative orientation of the LC material and light polarization. In actual working conditions, this orientation is continuously being varied by switching, thus making the LC more or less vulnerable to the UV light. Therefore, reliable tests of VAN LCOS UV aging must be performed on assembled devices that are continuously switched along the experiment. Performing this procedure in a climate chamber may be cumbersome as power supply for the test cells is required inside the chamber. Moreover, LCOS devices are expensive; therefore an alternative test procedure for material selection would be convenient.

As part of a study on UV aging of LCOS VAN devices, our group is testing a number of negative nematic LC materials to check whether they are suitable for high light flux projection applications. We have developed a simple method to test the UV photostability of the materials that avoids the use of LCOS backplane and switching. This method is used, along with electrooptical and dynamic tests, to select a reduced number of LC materials as candidates that eventually will be tested in fully assembled LCOS devices. 


\section{EXPERIMENTAL}

To avoid the UV filtering effect of the cell glass plates, LC samples are sandwiched between Spectrosil quartz plates. Homogenous and homeotropic $1.5 \mu \mathrm{m}$ cells have been prepared to account for unswitched and switched VAN samples respectively. In these conditions, the polarized UV spectrum will depend only on the LC material and the relative orientation of the LC and the light polarization.

Vertical alignment nematic (VAN) displays behave as linear retardation plates, the phase delay induced in the incoming light depending on the LC birefringence and the display thickness. When the phase retardation is $\pi$, the transmission of the VAN cell between crossed polarizers is maximum. Retardation is proportional to the difference in optical length of the display light path $(\Delta n \cdot d)$, where $\Delta n$ is the liquid crystal birefringence and $d$ is the thickness of the display. LCOS are reflective devices; hence light path is twice the transmissive path. Therefore the linear retardation is

$$
\Delta=\frac{4 \pi}{\lambda} \cdot \Delta n \cdot d
$$

Being linear retarders, VAN birefringence and thickness must be tuned up for maximum transmission of the ON state. Considering the yellow-green $550-590 \mathrm{~nm}$ spectral region as the reference wavelength, a $\Delta n \cdot d$ product of about $0.14 \mu \mathrm{m}$ is required for a full $\pi$ retardation. A few years ago, it was customary to use cell thicknesses about $2 \mu \mathrm{m}$ [7], thus requiring LCs with fairly low birefringence $(\sim 0.07)$ and low rotational viscosity as well. Improvements in materials design and manufacturing have reversed this trend. At present, thinner cells about $1-1.5 \mu \mathrm{m}$ are preferred to reduce relaxation time upon switching. Consequently, birefringence has to be increased up to $0.1-0.15$. In this work, two negative mixtures are used: the commercially available Merck MLC $7029(\Delta n=0.1265)$ and the experimental mixture $1754 \mathrm{C}$ prepared by the Military University of technology of Warsaw $(\Delta n=0.1498)$.

Measurements have been carried out in a UV-Vis dual channel absorption spectrometer provided with UV polarizers. Ultraviolet absorption spectra of the samples in the wavelength range 280-380 nm were obtained using a Perkin-Elmer Lambda 2 spectrophotometer. Incident light was polarized using Oriel ultraviolet linear dichroic polarizers (cut-off, $260 \mathrm{~nm}$ ). Background correction was performed by placing two quartz plates with the same alignment conditioning in the blank channel, the plates being not assembled in a cell to avoid Fabry-Perot interference fringes. 
The incident light of the spectrophotometer without polarizers is elliptically polarized, having its longer axis near the horizontal plane. Therefore, in order to keep constant the experimental setup, the relative sample orientation with light polarization was varied by rotating the samples, while maintaining the UV polarizers in the horizontal position.

It is worth mentioning that the spectrophotometer measurements were performed in transmissive mode since the instrument accuracy is far better than in reflective mode. This has no effect on the results of this work since we were just interested in absorbance and dichroism measurements. Yet thicker $(1.5 \mu \mathrm{m})$ samples have been used to account for the single light path.

\section{RESULTS AND DISCUSSION}

UV absorbance of the cells was measured in three different cases corresponding to xyz orientations of the indicatrix principal axis. Homeotropic samples were used to measure absorption with the material oriented parallel to the light propagation. Homogeneous samples were oriented parallel and perpendicular to the light polarization to account for the two remaining orientations. The setup intends to mimic a VAN cell being switched from its relaxed state, nearly perpendicular to the plates, to a switched state parallel to the plates (in an actual VAN cell, the switched state would be forming a $45^{\circ}$ angle with the polarization.

Typical results of these measurements are shown in Figure 1. As expected, the absorption spectrum of homeotropically aligned cells, which corresponds to the unswitched or OFF state of the VAN cell, shows a significantly lower optical density than the homogenously aligned cell when the orientation of the latter is parallel to the polarization of the impinging UV light. If the same homogenously aligned cell is rotated $90^{\circ}$, its absorption spectrum (not shown in the figure) matches approximately the homeotropic case. The results are compatible with a strong $\pi-\pi^{*}$ transition whose dipole moment is oriented parallel to the LC indicatrix.

From the practical point of view, these results can be used to evaluate LC materials as VAN candidates. In the cases shown in Figure 1, the optical density of the experimental mixture $1754 \mathrm{C} \mathrm{(Fig.} 1 a$ ) is lower than the one of the commercial Merck mixture MLC 7029 (Fig. 1b). Moreover, the UV absorption tail at longer wavelengths starts at $<320 \mathrm{~nm}$ in the $1754 \mathrm{C}$ case while at $>330 \mathrm{~nm}$ in the MLC 7029 case. As the impinging light in an actual projection engine features a UV cut-off filter, this tail may be responsible for the main fraction of the 


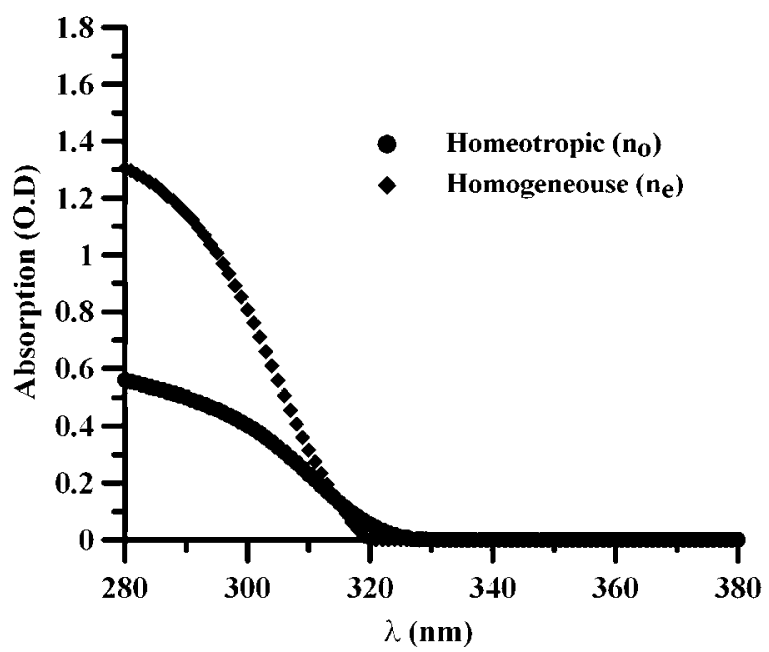

(a)

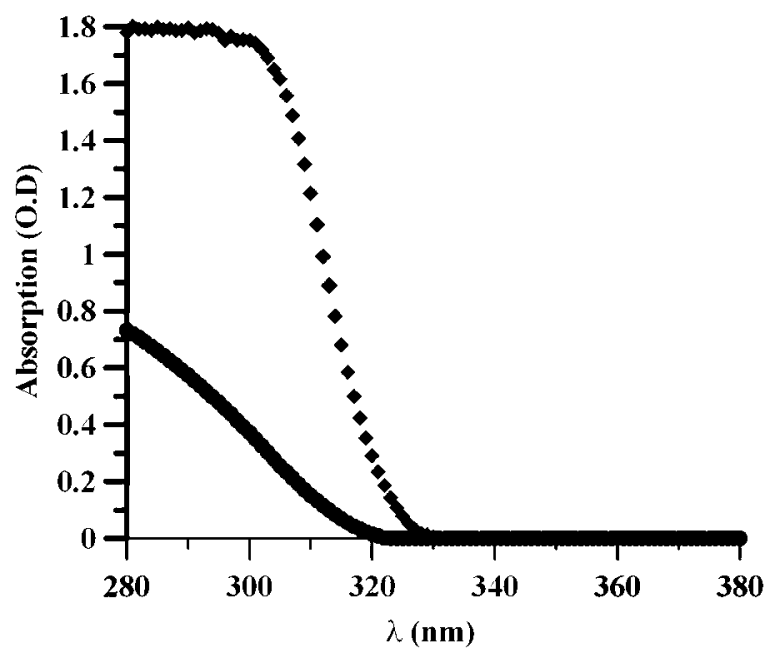

(b)

FIGURE 1 UV absorption dichroism spectrum using Perkin-Elmer spectrometer when the incident light. (a) Experimental mixture 1754 C, (b) Merck mixture MLC7029.

residual absorption, thus playing a role on the long-term photochemical stability of the material. Consequently, the experimental material $1754 \mathrm{C}$ shall be preferred according to this parameter. 
UV aging tests are just one of a number of characterization tests that are performed to assess the adequacy of a given LC material to the strict VAN LCOS requirements. Further electrooptic characterizations have been performed at $50^{\circ} \mathrm{C}$ (the expected LCOS working temperature) in a Mettler hot stage on a polarizing microscope. Figure 2 shows the dynamic grayscale of the experimental liquid crystal mixture $1754 \mathrm{C}$ (Fig. 2a) and the commercial liquid crystal mixture MLC 7029 (Fig. 2b) being driven with a $120 \mathrm{~Hz}$ frametime. The $10-90 \%$ dynamic range of the former is less than one half the range of the latter. The voltage increment required for the full grayscale to develop must be kept below a certain threshold $(4-5 \mathrm{~V})$ to allow the

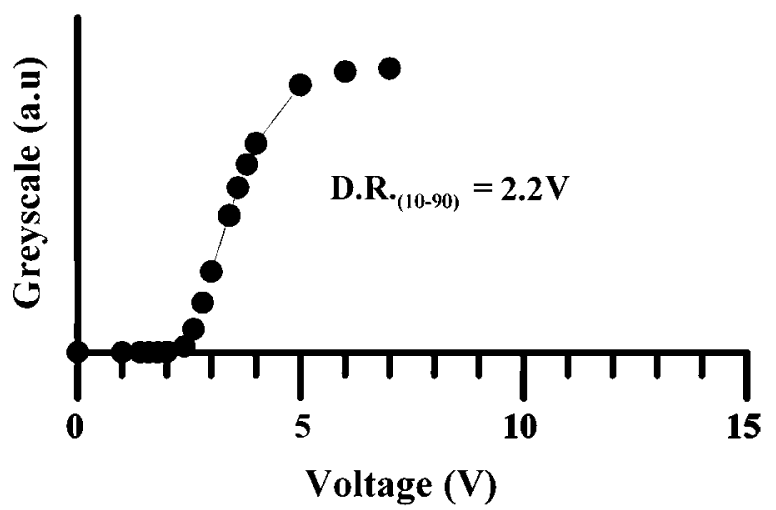

(a)

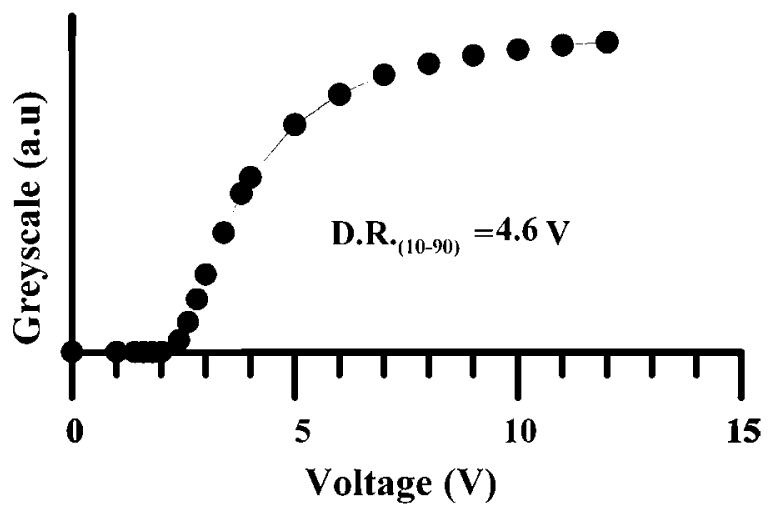

(b)

FIGURE 2 Gray scale transmission of an vertical aligned nematic cell filled with (a) Experimental mixture 1754 C, (b) Merck mixture MLC7029. 


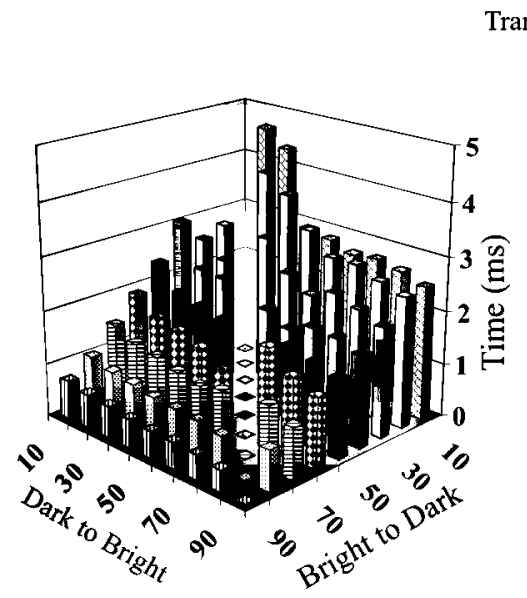

(a)

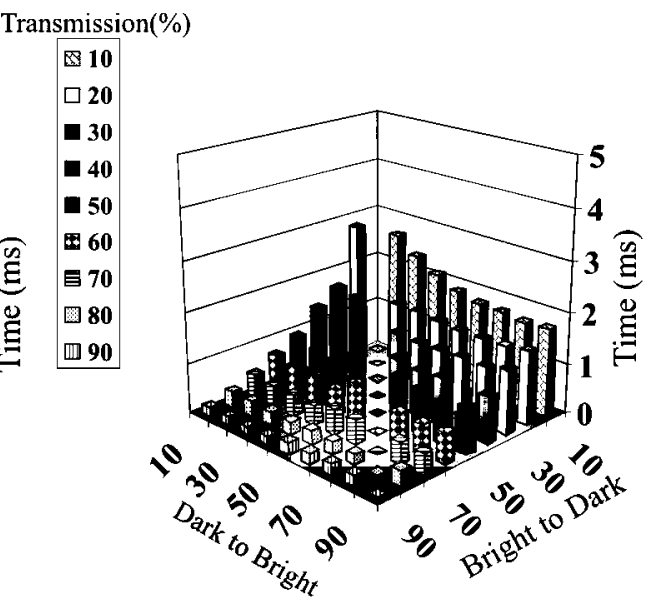

(b)

FIGURE 3 Response time between transmission levels at 10\% steps, in cells filled with (a) Experimental mixture 1754C, (b) Merck mixture MLC7029.

use of conventional CMOS electronics. Both materials meet this condition, although the commercial material is quite close to the limit.

Figure 3 shows a full characterization of gray to gray switching times taken in $10 \%$ increments. Switching times were lower than $3 \mathrm{~ms}$ in the MLC 7029 case and in most instances of the $1754 \mathrm{C}$ case. However, the time was higher in a few cases of the second material. Therefore, the commercial material performed better in this parameter. Improved (i.e., faster) experimental derivatives of $1754 \mathrm{C}$ mixture are presently under development.

\section{CONCLUSIONS}

The UV photochemical stability of VAN mixtures can be foreseen without using fully assembled devices by simulation the material switching with quartz cells having different induced alignments. The test is useful to select the most suitable candidates for the final device, although the test must be complemented with a number of characterization tests concerning their electrooptic and dynamic performance.

\section{REFERENCES}

Ong, H. L., Cheong, N., Lo, J., Metras, M., \& Woodard, O. (2003). SID'03, 680.

Bremer, M., Memmer, M. K. Paulutef, D., \& Tarumi, K. (2006). J. Soc. Informat.

Display, 14(6), 517. 
Yu, X. J. \& Kwok, H. S. (2006). Appl. Phys. Lett., 89, 031104.

Wen, C. H., Gauza, S., \& Wu, S. T. (2005). in Proc. SPIE, 5740, 67.

Lin, P. T., Wu, S. T., Shang, C. Y., \& Hsu, C. S. (2004). Mol. Cryst. Liq. Cryst., $411,243$.

Saleh, B. E. A. \& Teich, M. C. (1991). Fundamentals of Photonics. Wiley: New York. 